\title{
Association of PDCD1 polymorphisms with childhood-onset systemic lupus erythematosus
}

\author{
Rafael Velázquez-Cruz ${ }^{1}$, Lorena Orozco*,1, Francisco Espinosa-Rosales ${ }^{2}$, \\ Roberto Carreño-Manjarrez ${ }^{3}$, Eunice Solís-Vallejo ${ }^{4}$, Norma D López-Lara ${ }^{2}$, \\ Ivon K Ruiz-López ${ }^{5}$, Ana L Rodríguez-Lozano ${ }^{3}$, Jesús K Estrada-Gil ${ }^{1}$, \\ Gerardo Jiménez-Sánchez ${ }^{1}$ and Vicente Baca ${ }^{*}, 5$
}

\begin{abstract}
${ }^{1}$ Instituto Nacional de Medicina Genómica, Mexico City, Mexico; ${ }^{2}$ Instituto Nacional de Pediatría, Mexico City, Mexico; ${ }^{3}$ Hospital Infantil de México, Mexico City, Mexico; ${ }^{4}$ Hospital General Centro Médico 'La Raza', IMSS, Mexico City, Mexico; ${ }^{5}$ Hospital de Pediatría, Centro Médico Nacional Siglo XXI, IMSS, Mexico City, Mexico
\end{abstract}

A regulatory single nucleotide polymorphism (SNP) PD1.3G/A located on programmed cell death 1 (PDCD1) gene, was shown to be involved in susceptibility to systemic lupus erythematosus (SLE) in Swedish, European American, and Mexican cases. However, association to childhood-onset SLE has not been analyzed. The aim of this study was to investigate the association of $P D C D 1$ polymorphisms and haplotypes with susceptibility to childhood-onset SLE in Mexican population. Three PDCD1 SNPs, PD1.3G/A, PD1.5C/T, PD1.6G/A, were analyzed in $\mathbf{2 5 0}$ childhood-onset SLE Mexican patients and 355 healthy controls in a case-control association study. Polymorphisms were genotyped by TaqMan technology. Stratification analysis was performed on the SLE cohort to investigate the SNP association with renal disorder. In addition, haplotypes were constructed with these three SNPs. The PD1.3A allele was significantly associated to childhood-onset SLE $(P=0.0019$, odds ratio (OR) $2.73,95 \%$ confidence interval $(95 \% \mathrm{CI}) 1.35-5.56)$. The other PDCD1 SNPs did not show association. A total of 155 patients $(62 \%)$ had nephritis, and no association was observed with PDCD1 SNPs. The ACG haplotype (PD1.3A, PD1.5C, PD1.6G) included almost all PD1.3A alleles, and it was more frequent in SLE patients $(5.5 \%)$ than in controls $(2.1 \%)(P=0.003$; OR $2.73,95 \% \mathrm{Cl} 1.37-5.46)$. The haplotype structure in Mexican controls was significantly different from those reported in Spanish and Swedish. Our results support association of the PD1.3A SNP to susceptibility of childhood-onset SLE in Mexican population and does not show association to lupus nephritis in this age group.

European Journal of Human Genetics (2007) 15, 336-341. doi:10.1038/sj.ejhg.5201767; published online 17 January 2007

Keywords: systemic lupus erythematosus (SLE); pediatric rheumatology; SNPs

*Correspondence: Dr L Orozco, Instituto Nacional de Medicina Genómica, Periférico Sur 4124, Torre Zafiro II, $6^{\circ}$ piso, Col. Jardínes del Pedregal, México, DF, CP 01900, Mexico.

Tel: + 52555350 1957, Fax: + 52555350 1968,

E-mail: lorozco@inmegen.gob.mx or Dr V Baca, Departamento de Reumatología, Hospital de Pediatría, Centro Médico Nacional Siglo XXI, IMSS. Ave. Cuauhtémoc No. 330, Col. Doctores, CP 06720, México, DF, Mexico.

Tel: + 52555627 6900, Ext. 22457, Fax: + 52555671 0258,

E-mail: vicbaca@prodigy.net.mx

This work was submitted in partial fulfilment of the requirements for the PhD degree in for RVC at Doctorado en Ciencias Biomédicas, Universidad Nacional Autónoma de México.

Received 25 September 2006; revised 21 November 2006; accepted 22 November 2006; published online 17 January 2007

\section{Introduction}

Systemic lupus erythematosus (SLE) is a complex autoimmune disorder that predominantly affects women during childbearing age. ${ }^{1}$ However, $15-17 \%$ of all SLE patients present in children younger than age $16 .^{2}$ Although clinical manifestations and immunological findings of pediatric SLE are similar to those seen in adults with SLE, in childhood-onset SLE the initial symptoms tend to be more severe and it has a more aggressive clinical course. ${ }^{3-5}$

Susceptibility to SLE has been attributed to complex interaction between genetic and environmental factors. In 
recent years, several potential SLE susceptibility loci have been identified by nine complete genome scans using family collections that differ greatly in ethnic compositions and geographic locations, although only eight of these have been significantly linked to SLE. These map to 1q23, 1q25-31, 1q41-42, 2q35-37, 4p16-15.2, 6p11-21, $12 \mathrm{q} 24$ and $16 \mathrm{q} 12 .{ }^{6}$ Recently, Prokunina et al found the programmed cell death gene (PDCD1) within the 2q37 region as a good candidate risk gene for SLE. ${ }^{7}$ PDCD1 encodes an inhibitory immune receptor (PD-1), member of the immunoglobulin superfamily that carries an immune tyrosine-based inhibitory motif (ITIM), and plays a pivotal role in maintaining peripheral immune tolerance. ${ }^{8} \mathrm{C} 57 \mathrm{BL} /$ 6 background mice deficient in PD-1 expression develop a lupus-like syndrome, with glomerulonephritis and arthritis. ${ }^{9}$ Prokunina et al identified seven single nucleotide polymorphisms (SNPs) within the PDCD1 gene. One of them (PD1.3 G/A), a regulatory SNP located in intron 4, showed to be involved in susceptibility to SLE in Swedish, European American, and Mexican families and in sporadic cases. ${ }^{7}$ However, other studies that included sporadic SLE patients from northern Sweden and Denmark did not find association to PD1.3A SNP. ${ }^{10,11}$ Moreover, the PD1.3A allele has been associated to renal manifestations in Swedish patients with SLE, but not in female patients from the United States with European American descent. ${ }^{10,12}$ In contrast, analysis of a large Spanish cohort showed that the PD1.3G allele is associated with susceptibility to SLE as opposed to the observed for other populations. ${ }^{13}$

We performed a case-control study to test the association between SNPs on PDCD1 gene and susceptibility to childhood-onset SLE in a cohort of Mexican patients.

\section{Patients and methods \\ Patients}

We studied 250 unrelated patients with childhood-onset SLE, consisting of 228 cases with sporadic SLE and 22 cases with familial SLE who were recruited from four pediatric hospitals in Mexico City: Hospital de Pediatría Centro Médico Nacional Siglo XXI, Instituto Nacional de Pediatría, Hospital Infantil de México, and Hospital General Centro Médico 'La Raza'. All patients were $<16$ years of age at onset of disease and fulfilled the American College of Rheumatology (ACR) criteria for the diagnosis of SLE. ${ }^{14}$ Of these, 214 were female (85.6\%) and 36 were male $(14.4 \%)$, with a mean \pm SD age at onset of $11.62 \pm 2.46$ years in the whole group. All patients were born in Mexico like their parents, grandparents and great-grandparents. Additionally, 355 blood bank donors were included as healthy control group. The controls were drawn from Mexico City and sex matched, consisting of 305 female subjects (86\%) and 50 male subjects (14\%). This study was approved by the respective local ethics and research committees and all individuals signed an informed consent.

\section{Study design}

We performed a case-control association study to investigate whether the PDCD1 polymorphisms were associated with childhood-onset SLE. We studied three of the seven PDCD1 SNPs previously described by Prokunina et al not fully linked to each other: PD1.3 (7,146G/A), PD1.5 $(7,785 \mathrm{C} / \mathrm{T})$, and PD1.6 (8,738G/A). ${ }^{7}$ We also constructed haplotypes with the alleles of these three SNPs.

Stratification analysis was performed in the SLE cohort to investigate the SNP association with renal disorder. One hundred and fifty five patients (62\%) were considered as having lupus nephritis according to the ACR criteria and were confirmed by kidney biopsy in 104 (67\%). Seventeen were Class II (16\%), seven Class III (7\%), seventy six Class IV (73\%), three Class V (3\%), and one Class VI (1\%), according to the World Health Organization (WHO) classification. $^{15}$

\section{Genotyping}

Genomic DNA from patients and controls was extracted from peripheral blood leukocytes using the conventional technique of phenol chloroform ${ }^{16}$ or the GenePure Genomic Blood DNA Purification kit (Gentra Systems, Minneapolis, MN, USA) according to the manufacturer's protocol. PD1.3, PD1.5, and PD1.6 polymorphisms were genotyped using the TaqMan system $5^{\prime}$-allele discrimination Assay-By-Design method (Applied Biosystems, Foster City, CA, USA). The primers and probes used for identification of each allele are shown in Table 1.

Polymerase chain reaction (PCR) was carried out by using TaqMan Universal Master Mix (Applied Byosystems) in $5 \mu$ final volume reactions, using $20 \mathrm{ng}$ of genomic DNA.

Table 1 The $5^{\prime}$ nuclease assays (TaqMan) for PDCD1 polymorphisms

\begin{tabular}{|c|c|}
\hline \multicolumn{2}{|l|}{ PD1.3 } \\
\hline $\begin{array}{l}\text { Forward-primer } \\
\text { Reverse-primer } \\
\text { G-allele probe } \\
\text { A-allele probe }\end{array}$ & $\begin{array}{l}\text { 5'-CCTCAATCCCTAAAGCCATGATCTG-3' } \\
\text { 5'-CAGGCAGGCACACACATG-3' } \\
\text { 5'-ACCTGCGGTCTCC-MGB }{ }^{\prime}-\mathrm{NFQ}^{\mathrm{b}}-3^{\prime} \\
\text { 5'-ACCTGCAGTCTCC-MGB }{ }^{\mathrm{a}}-\mathrm{NFQ}^{\mathrm{b}}-3^{\prime}\end{array}$ \\
\hline \multicolumn{2}{|l|}{ PD1.5 } \\
\hline $\begin{array}{l}\text { Forward-primer } \\
\text { Reverse-primer } \\
\text { C-allele probe } \\
\text { T-allele probe }\end{array}$ & $\begin{array}{l}\text { 5'-GCGGAATGGGCACCTCAT-3' } \\
\text { 5'-TCCATCCTCAGGCCTCAGT-3' } \\
\text { 5'-CGTCGGCTGAGCC-MGB }{ }^{\prime}-\mathrm{NFQ}^{\mathrm{b}}-3^{\prime} \\
5^{\prime}-\text {-CCGTCAGCTGAGCC -MGB }{ }^{\mathrm{a}}-\mathrm{NFQ}^{\mathrm{b}}-3^{\prime}\end{array}$ \\
\hline \multicolumn{2}{|l|}{ PD1.6 } \\
\hline $\begin{array}{l}\text { Forward-primer } \\
\text { Reverse-primer } \\
\text { G-allele probe } \\
\text { A-allele probe }\end{array}$ & 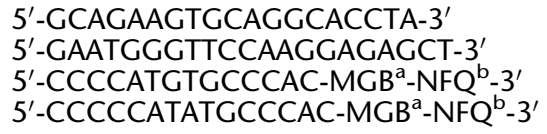 \\
\hline
\end{tabular}

Primers and probes were designed using Assays-by-Design (Applied Biosystems).

The probes were labeled at their $5^{\prime}$ ends with FAM $^{\mathrm{TM}}$ (the first allele) and $\mathrm{VIC}^{\mathrm{TM}}$ (the second allele) and the $3^{\prime}$ ends contained quenchers.

${ }^{\mathrm{a}}$ Minor Groove Binder.

${ }^{\mathrm{b}}$ Quencher. 
Thermal cycling conditions were $95^{\circ} \mathrm{C}$ for $10 \mathrm{~min}$, followed by 40 cycles of $92^{\circ} \mathrm{C}$ for $15 \mathrm{~s}$ and $60^{\circ} \mathrm{C}$ for $1 \mathrm{~min}$. The genotype was automatically attributed in each sample measuring the allele-specific fluorescence in the ABI Prism 7900 HT Sequence Detection System, using the SDS 2.2.2 software for allele discrimination (Applied Biosystems). The overall genotype call rate by TaqMan was 98\%; five samples from a total of 255 cases and five samples from a total of 360 controls were discarded from the analysis because genotyping was not successful in one or more of the SNPs analyzed. The assay reproducibility was 100\% in the 250 repeated samples from patients and the 355 samples from controls.

To confirm the genotype obtained by the TaqMan $5^{\prime}$ allele discrimination assay, PCR-restriction fragment length polymorphism (PCR-RFLP) was performed in 201 samples from cases (80\%), and 260 samples from controls (73\%). The PD1.5 and PD1.6 SNPs were genotyped by this technique as previously described. ${ }^{13}$ The PCR-RFLP on PD1.3 was performed in a $25 \mu \mathrm{l}$ reaction mixture containing $100 \mathrm{ng}$ of genomic DNA, $100 \mu \mathrm{M}$ of dNTPs, $0.1 \mu \mathrm{M}$ of each primer, $5 \mathrm{U}$ of Amplitaq Gold DNA polymerase (Applied Biosystems, USA), $1.5 \mathrm{mM}$ of $\mathrm{MgCl}_{2}$ and $1 \times \mathrm{PCR}$ buffer. Thermal cycling conditions were $95^{\circ} \mathrm{C}$ for $10 \mathrm{~min}$, followed by 35 cycles of $95^{\circ} \mathrm{C}$ for $15 \mathrm{~s}, 60^{\circ} \mathrm{C}$ for $30 \mathrm{~s}$, and $72^{\circ} \mathrm{C}$ for $15 \mathrm{~s}$. A final extension at $72^{\circ} \mathrm{C}$ for $5 \mathrm{~min}$ was carried out. The 180-pb PCR product was digested with $5 \mathrm{U}$ of Pst I (Invitrogen, CA, USA) at $37^{\circ} \mathrm{C}$ overnight and was resolved on a $15 \%$ polyacrylamide gel with ethidium bromide. The A allele include a Pst site that is not present in the $G$ allele. Therefore, digestion resulted in 130- and 50-bp fragments for the A allele or 180-bp fragments for the $\mathrm{G}$ allele. The primers sequences are $5^{\prime}$-CCCCAGGCAG CAACCTCAAT-3' and reverse primer: 5'-GACCGCAGG CAGGCACATAT-3', (conditions provided by AlarconRiquelme ME).

We also confirmed the genotype of PD1.3 SNP by sequencing on representative samples from each genotype. The system used for sequencing PD1.3 was the BigDye Ready Reaction Kit (Applied Biosystems) in an ABI Prism $3130 \mathrm{xL}$ Genetic Analyzer (Applied Biosystems). The genotype obtained by TaqMan was confirmed by PCRRFLP and by sequencing in $100 \%$ of the samples tested.

\section{Statistical analysis}

We used $\chi^{2}$ analysis to evaluate the significance of differences in genotype and allele frequencies between cases and controls. Also, allele frequencies for cases and controls were used to calculate the odds ratio (OR) and the $95 \%$ confidence interval $[95 \% \mathrm{CI}]$. The $\chi^{2}$ value was calculated from $2 \times 2$ contingency tables, and $P$-values were determined using one degree of freedom. The software used for all statistical calculations was the StatCalc program (Epi Info 2005 v3.3.2; Centers of Disease Control and Prevention, Atlanta, GA, USA). Genotype distributions in patients and controls were evaluated for departure from Hardy-Weinberg equilibrium by the FINETTI program using the exact test (http://ihg.gsf.de/cgi-bin/hw/hwa1.pl).

In the case-control haplotype analysis, Haploview v3.2 software was used to estimate haplotypes frequencies, which uses an implementation of the expectationmaximization algorithm. ${ }^{17}$ We tested association with $\chi^{2}$ analysis for each possible combination of haplotypes derived from the three SNPs (PD1.3, PD1.5 and PD1.6). In order to obtain a measure of significance corrected for multiple testing bias, we ran 10000 permutations to compute $P$-values using the Haploview program. $P$-values less than 0.05 were considered statistically significant.

\section{Results}

Genotype frequencies of PD1.3, PD1.5 and PD1.6 SNPs were found to be in Hardy-Weinberg equilibrium in cases $(P=1.0,0.58$ and 1.0$)$ and controls $(P=1.0,0.36$ and 0.33$)$, respectively. When genotype frequencies were compared between cases and controls only PD1.3 SNP showed statistically significant association, the heterozygous genotype GA was more frequent in patients with SLE than in controls (10.4 versus 4\%; $P=0.0016$; OR 2.83, 95\% CI 1.38-5.84) (Table 2). The PD1.3A allele frequency was 5.2\% in SLE patients while in controls it was $2 \%(P=0.0019$, OR $2.73,95 \%$ CI 1.35-5.56) (Table 3). When we analyzed only female cases and female controls as reported by Prokunina et $a l^{7}$ the association with PD1.3A allele was still significant $(P=0.0052$, OR 2.53, 95\% CI 1.29-4.95) (data not shown). The frequency of PD1.3A allele was lower in male SLE patients than female cases (2.8 versus $5.6 \%)$, and in male controls it was $0 \%$, whereas in female controls it was $2.3 \%$. Nevertheless, these differences could be due to the small number of male patients with SLE and male controls analyzed (36 and 50 respectively). Allele frequencies of PD1.5C and of PD1.6A polymorphisms did not differ significantly between cases and controls (Table 3 ).

A total of 155 patients (62\%) had nephritis according to biopsy and/or for ACR criteria. When association was evaluated between the PD1.3A SNP and glomerulonephritis, no significant differences were observed between patients with and those without lupus nephritis (5.48 and $4.73 \%$ respectively, $\left.\chi^{2}=0.133, P=0.71\right)$. Neither PD1.5C nor PD1.6A showed association to glomerulonephritis (data not shown).

Haplotype frequencies were estimated in female cases and in female controls using the three SNPs of PDCD1. A total of six haplotypes were identified, but only five had a frequency greater than $1 \%$ (Table 4 ). The ACG haplotype (PD1.3A, PD1.5C, PD1.6G) included almost all PD1.3A alleles, and it was more frequent in SLE patients (5.5\%) than in controls $(2.1 \%)(P=0.003$; OR $2.73,95 \%$ CI 1.37-5.46) (Table 4). Haplotype frequencies obtained in 
Table 2 Genotype distribution of PDCD1 polymorphisms in Mexican childhood-onset SLE

\begin{tabular}{lccrr}
\hline Genotype & SLE $\mathrm{n}=250(\%)$ & Controls $\mathrm{n}=355(\%)$ & $\mathrm{P}^{\mathrm{a}}$ & OR (95\% CI) \\
\hline PD1.3GG & $224(89.6)$ & $341(96)$ & 0.0016 & $0.35(0.17-0.72)$ \\
PD1.3GA & $26(10.4)$ & $14(4)$ & 0.0016 & $2.83(1.38-5.84)$ \\
PD1.3AA & $0(0)$ & $0(0)$ & 0.72 & $0.94(0.67-1.33)$ \\
PD1.5CC & $102(40.8)$ & $150(42)$ & 0.34 & $1.17(0.84-1.64)$ \\
PD1.5CT & $119(47.6)$ & $155(43.7)$ & 0.37 & $0.80(0.48-1.34)$ \\
PD1.5TT & $29(11.6)$ & $50(14.1)$ & 0.61 & $1.11(0.73-1.68)$ \\
PD1.6GG & $55(22)$ & $72(20.3)$ & 0.39 & $1.15(0.82-1.61)$ \\
PD1.6GA & $125(50)$ & $165(46.5)$ & 0.17 & $0.78(0.54-1.13)$ \\
PD1.6AA & $70(28)$ & $118(33.2)$ & & \\
\hline
\end{tabular}

$\mathrm{SLE}=$ systemic lupus erythematosus; $\mathrm{OR}=$ odds ratio; $95 \% \mathrm{Cl}=95 \%$ confidence interval.

${ }^{\text {a }} P$-value uncorrected for multiple tests, 1 degree of freedom.

Table 3 Distribution of alleles of SNPs in PDCD1 gene in Mexican childhood-onset SLE

\begin{tabular}{lccrr}
\hline Allele & SLE $n / N^{\mathrm{a}}(\%)$ & Controls $n / N^{\mathrm{a}}(\%)$ & $\mathrm{P}^{\mathrm{b}}$ & $O R(95 \% \mathrm{Cl})$ \\
\hline PD1.3A & $26 / 500(5.2)$ & $14 / 710(2.0)$ & $0.0019^{\mathrm{c}}$ & $2.73(1.35-5.56)$ \\
PD1.5C & $323 / 500(64.6)$ & $455 / 710(64)$ & 0.85 & $1.02(0.88-1.35)$ \\
PD1.6A & $265 / 500(53)$ & $401 / 710(56.5)$ & 0.23 & $0.87(0.69-1.10)$ \\
\hline
\end{tabular}

${ }^{a} \mathrm{n} / \mathrm{N}=$ number of alleles out of total number of chromosomes. $\mathrm{SLE}=$ systemic lupus erythematosus; $\mathrm{OR}=$ odds ratio; $95 \% \mathrm{Cl}=95 \%$ confidence interval.

${ }^{\mathrm{b}} P$-value uncorrected for multiple tests, 1 degree of freedom.

${ }^{\mathrm{C}} P=0.0089$ after correcting the $P$-value for multiple testing by Haploview program using 10000 permutations.

Table 4 Haplotype analysis of PDCD1 polymorphisms in Mexican SLE female cases and female controls

\begin{tabular}{lccccccc}
\hline Haplotype number & PD1.3 & PD1.5 & PD1.6 & Cases $\mathrm{n}^{\mathrm{a}}=428$ & Frequency & ${\text { Controls } \mathrm{n}^{\mathrm{a}}=610}^{\text {Frequency }}$ \\
\hline 1 & G & C & A & 123 & 0.29 & 193 & 0.32 \\
2 & G & C & G & 125 & 0.29 & 179 & 0.29 \\
3 & G & T & A & 106 & 0.25 & 0.27 \\
4 & G & T & G & 50 & 0.12 & 0.094 \\
$5^{\text {b }}$ & A & C & G & 24 & 0.055 & 137 & 0.021 \\
6 & A & C & A & 0.3 & 0.0007 & 1.2 & 0.002 \\
\hline
\end{tabular}

a Number of studied chromosomes.

${ }^{\mathrm{b}} P=0.003$, uncorrected for multiple tests; odds ratio $=2.73 ; 95 \%$ confidence interval: $1.37-5.46 . P=0.045$ after correcting the $P$-value for multiple testing by Haploview program using 10000 permutations.

female Mexican controls were compared to those previously reported in female controls from Spain and Sweden. ${ }^{7,13}$ We observed significant differences between these populations and the Mexican (Figure 1). Interestingly, the GCA haplotype, which was the least frequent in Spanish (1\%) and Swedish controls (2\%), resulted the most frequent in Mexican controls (32\%) $\left(P<1.0 \times 10^{-7}\right.$ and $P<4.0 \times 10^{-7}$, respectively). The GTA haplotype showed to be more frequent in Mexican (27\%) than in Spanish $(9.67 \%)$ or Swedish populations $(19 \%)$, however, this difference showed significance only when compared to Spanish $\left(P<1.0 \times 10^{-7}\right)$. On the other hand, haplotype frequencies of GCG, GTG, and ACG were lower in Mexican population than in Spanish or Swedish female controls. These differences were statistically significant, except for the ACG haplotype between Mexican and Swedish (Figure 1).

\section{Discussion}

The genetic basis in childhood-onset SLE is currently unclear. Although several new insights have been obtained in the past decade using linkage and association studies of multicases SLE families as well as case-control studies, most of these studies have involved mainly patients with adult-onset SLE. Recently, we have replicated the observed association of the PTPN22 gene with adult-onset SLE in childhood-onset SLE in Mexican population, ${ }^{18}$ and to our knowledge there are only two other genetic studies with a small sample size on pediatric SLE. ${ }^{19,20}$ One was performed in 46 Chinese children showing association between SLE and a SNP in RANTES gene. ${ }^{19}$ The other one found an association of polymorphisms in the estrogen receptor $\alpha$ gene in 41 Korean with childhood-onset SLE. ${ }^{20}$

We performed a case-control study to test association between SNPs on the PDCD1 gene to childhood-onset SLE 


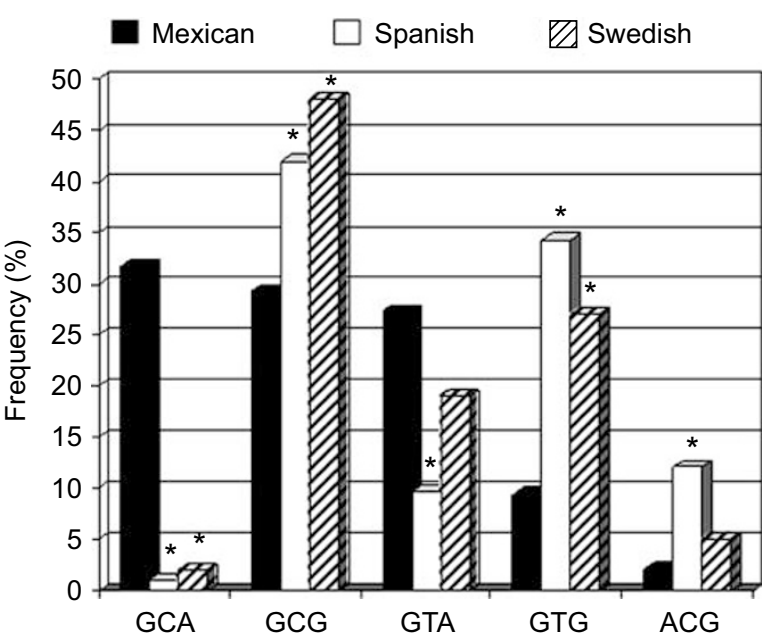

Figure 1 Haplotype frequencies of $P D C D 1$ derived from PD1.3, PD1.5, and PD1.6 SNPs in female controls from Mexico, Spain and Sweden. Haplotype frequencies for the Mexican female controls were estimated from 610 chromosomes. Haplotype frequencies for the Spanish female controls and Swedish female controls were obtained from Figure 1 of reference. ${ }^{13}{ }^{*} P<0.05$ versus Mexican female controls.

in a large cohort of Mexican patients. Prokunina et $a l^{7}$ previously reported an association of the PD1.3A allele with SLE in adults from three different populations, including Mexicans. This SNP disrupts the predicted DNA-binding site for the runt-related transcription factor 1 (RUNX1) located in an intronic enhancer, suggesting a mechanism through which it can contribute to the development of SLE in humans. ${ }^{7}$ Our results show that the PD1.3A allele increases genetic risk to childhood-onset SLE in Mexicans. However, we found a weaker association of this SNP with childhood-onset SLE female patients $(P=0.0052$, OR $2.53,95 \%$ CI $1.29-4.95)$ compared with that reported by Prokunina et $a{ }^{7}{ }^{7}$ in Mexican female adults with SLE $(P=0.0023$, OR $3.23,95 \%$ CI $1.46-7.16$; data calculated from Prokunina $\mathrm{et}^{\mathrm{Cl}}$ ).

As renal disease is extremely common in childhoodonset SLE, and it has been previously associated with the PD1.3A allele in adult SLE Swedish population, we explored the possible association between this SNP with lupus nephritis in Mexican children with SLE. Despite a high frequency of lupus nephritis (62\%) in our sample, we could not confirm association of PD1.3A allele with the phenotype. No association with renal disorder was also found in female patients with SLE from the United States of European American descent. ${ }^{12}$ This discrepancy could be explained by genetic heterogeneity.

Pediatric SLE is phenotypically similar to adult SLE, however childhood-onset SLE has a higher incidence of renal, neurological, hematological, cardiac and pulmonary disease, resulting in to more severe presentation and aggressive clinical course. ${ }^{3-5}$
The earlier-onset and the higher severity of the disease in childhood-onset than in adult-onset SLE, could be explained because of the presence of different polymorphisms or combination of them, or because there is a greater genetic background of susceptibility alleles in the childhood-onset SLE.

Mexican mestizos are an admixed population with a complex genetic structure, where Native American genes account for 51\%, European genes for $45.4 \%$, and African genes for $3.7 \% .^{21}$ This could explain the observed difference in the distribution of haplotype frequencies between Mexican population and Spanish and Swedish populations. The high frequency of the GCA haplotype in Mexican population compared with Spanish and Swedes (32 versus 1 and $2 \%$, respectively) suggests that in our population this haplotype could be provided from Amerindian ancestors. However, further studies on Mexican Amerindians are needed to confirm this asseveration.

In conclusion, our results support the involvement of the PD1.3A SNP as a genetic risk factor for susceptibility in childhood-onset SLE in Mexican population but not for lupus nephritis. Further studies comparing the genetic background between childhood-onset and adult-onset SLE patients in ethnically homogeneous populations would allow us to explain the phenotypical differences between these populations.

\section{Acknowledgements}

We thank Martha Alarcón-Riquelme and Antonio González, for providing the haplotype frequencies of PDCD1 from the Swedish and Spanish female controls. Supported by grants from the Consejo Nacional de Ciencia y Tecnología (CONACYT: SALUD-2004-01153) and by the Instituto Mexicano del Seguro Social, Fondo para el Fomento a la Investigación Médica (FOFOI: FP-2003/014).

\section{References}

1 Wallace DJ: The clinical presentation of systemic lupus erythematosus; In: Wallace DJ, Hahn BH (eds).: Dubois' Lupus Erythematosus. 6th ed. Philadelphia: Lippincott, Williams \& Wilkins, 2002, pp 621-628.

2 Singsen BH: Rheumatic diseases of childhood. Rheum Dis Clin North Am 1990; 16: 581-599.

3 Glidden RS, Mantzouranis E, Borel Y: Systemic lupus erythematosus in childhood: clinical manifestations and improved survival in fifty-five patients. Clin Immunol Immunopathol 1983; 29: $196-210$.

4 King KK, Kornreich HK, Bernstein BH, Singsen BH, Hanson V: The clinical spectrum of systemic lupus erythematosus in childhood. Arthritis Rheum 1977; 20: 287-294.

5 Meislin AG, Rothfield N: Systemic lupus erythematosus in childhood: analysis of 42 cases with comparative data in 200 adult cases followed concurrently. Pediatrics 1968; 42: 37-49.

6 Tsao BP: Update on human systemic lupus erythematosus genetics. Curr Opin Rheumatol 2004; 16: 513-521.

7 Prokunina L, Castillejo-Lopez C, Oberg F et al: A regulatory polymorphism in PDCD1 is associated with susceptibility to systemic lupus erythematosus in humans. Nat Genet 2002; 32: 666-669. 
8 Nishimura H, Honjo T: PD-1: an inhibitory immunoreceptor involved in peripheral tolerance. Trends Immunol 2001; 22: $265-268$.

9 Nishimura H, Nose M, Hiai H, Minato N, Honjo T: Development of lupus like autoimmune diseases by disruption of the PD-1 gene encoding an ITIM motif-carrying immunoreceptor. Immunity 1999; 11: $141-151$.

10 Johansson M, Arlesting L, Moller B, Rantapaa-Dahlqvist S: Association of a PDCD1 polymorphism with renal manifestations in systemic lupus erythematosus. Arthritis Rheum 2005; 52: $1665-1669$.

11 Nielsen C, Laustrup H, Voss A, Junker P, Husby S, Lillevang ST: A putative regulatory polymorphism in PD-1 is associated with nephropathy in a population-based cohort of systemic lupus erythematosus patients. Lupus 2004; 13: 510-516.

12 Prokunina L, Gunnarsson I, Sturfelt G et al: The systemic lupus erythematosus-associated PDCD1 polymorphism PD1.3A in lupus nephritis. Arthritis Rheum 2004; 50: 327-328.

13 Ferreiros-Vidal I, Gomez-Reino JJ, Barros F et al: Association of PDCD1 with susceptibility to systemic lupus erythematosus: evidence of population-specific effects. Arthritis Rheum 2004; 50: 2590-2597.

14 Tan EM, Cohen AS, Fries JF et al: The 1982 revised criteria for the classification of systemic lupus erythematosus. Arthritis Rheum 1982; 25: $1271-1277$.
15 Churg J, Bernstein J, Glassock RJ: Renal Disease: Classification and Atlas of Glomerular Diseases, 2nd edn New York, Tokyo: Igaky-Shoin, 1995.

16 Blin N, Sttaford D: A general method for isolation of high molecular weigh DNA from eukaryotes. Nucleic Acid Res 1976; 3: 2303-2308

17 Barret JC, Fry B, Maller J, Daly MJ: Haploview: analysis and visualization of LD and haplotype maps. Bioinformatics 2005; 21 : 263-265.

18 Baca V, Velázquez-Cruz R, Salas-Martínez G, Espinosa-Rosales F Saldaña-Alvarez Y, Orozco L: Association analysis of the PTPN22 gene in childhood-onset systemic lupus erythematosus in Mexican population. Genes Immun 2006; 7: 693-695.

19 Liao $\mathrm{CH}$, Yao TC, Chung HT, See LC, Huo ML, Huang JL: Polymorphisms in the Promoter region of RANTES and the regulatory region of monocyte chemoattractant protein-1 among Chinese children with systemic lupus erythematosus. J Rheumatol 2004; 10: 2062-2067.

20 Lee YJ, Shin KS, Kang SW et al: Association of the oestrogen receptor alpha gene polymorphisms with disease onset in systemic lupus erythematosus. Ann Rheum Dis 2004; 10: 1244-1249.

21 Choudhry S, Coyle NE, Hua Tang H et al: Population stratification confounds genetic association studies among Latinos. Hum Genet 2006; 118: 652-664. 